Scientia Marina 75(2)

June 2011, 341-350, Barcelona (Spain)

ISSN: 0214-8358

doi: 10.3989/scimar.201175n2341

\title{
Biodiversity of deep-sea demersal megafauna in western and central Mediterranean basins
}

\author{
SAMUELE TECCHIO, EVA RAMÍREZ-LLODRA, FRANCISCO SARDÀ \\ and JOAN BAPTISTA COMPANY \\ Institut de Ciències del Mar (CSIC). Passeig Marítim de la Barceloneta 37-49, 08003 Barcelona, Spain. \\ E-mail: tecchio@icm.csic.es
}

\begin{abstract}
SUMMARY: Abundance, biomass and diversity patterns of bathyal and abyssal Mediterranean megafauna (fishes and invertebrates) were analyzed in the western Balearic Sea, the western Ionian Sea and the eastern Ionian Sea. Sampling was conducted with a Otter-trawl Maireta System (OTMS) at depths ranging from 600 to $4000 \mathrm{~m}$. A series of ecological indicators were computed: total abundance and biomass, Margalef species richness, Shannon-Wiener diversity and Pielou's index of evenness. A multidimensional scaling was applied, indicating that the megafauna communities were grouped by depth, while geographic area had a less defined influence. Margalef richness declined with depth in all three areas, but more steeply in the western Ionian Sea. Pielou's evenness behaved differently in the three zones, showing a V-shaped curve in the eastern Ionian while showing a decreasing pattern in the other two areas. At lower slope depths, massive presence of the fishes Alepocephalus rostratus in the western basin and Bathypterois mediterraneus in the central basin caused a sharp reduction in evenness.
\end{abstract}

Keywords: diversity, megafauna, deep sea, western Mediterranean, western Ionian, eastern Ionian.

\begin{abstract}
RESUMEN. PATRones DE BIODIVERSIDAd DE LA MEgafaUna DEMERSAL DEL MAR PROFUNDO EN LAS CUENCAS OCCIDENTAl y CENTRAl del MediterRáneO. - Se analizaron los patrones de abundancia, biomasa y diversidad de la megafauna (peces y invertebrados) batial y abisal del Mediterráneo. El muestreo se llevó a cabo con el Sistema de Arrastre Maireta a profundidades entre 600 y 4000 m, en el mar Balear occidental, el mar Jónico occidental y el mar Jónico oriental. Por cada pesca, se calculó una serie de indicadores ecológicos, después de estandardizar por el área de barrido: abundancia y biomasa totales, riqueza específica de Margalef, diversidad de Shannon-Wiener y el índice de equitatividad de Pielou. Se aplicó un multidimensional scaling (MDS) que indicó un agrupamiento de las comunidades de megafauna por profundidad, mientras que el área geográfica tiene una influencia menos definida. El índice de riqueza específica de Margalef disminuye con la profundidad en las tres áreas, pero de manera más pronunciada en el Mar Jónico occidental. La equitatividad de Pielou se comporta de manera diferente en las tres zonas, presentando una curva en forma de $\mathrm{V}$ en el Jónico oriental, y un patrón decreciente en las otras dos áreas. En el talud continental inferior, la presencia masiva de los peces Alepocephalus rostratus en la cuenca occidental y Bathypterois mediterraneus en la zona central causan una reducción drástica de la equitatividad.
\end{abstract}

Palabras clave: diversidad, megafauna, mar profundo, Mediterráneo occidental, Jónico occidental, Jónico oriental.

\section{INTRODUCTION}

Deep-sea ecosystems are known to be the largest repository of biodiversity of the biosphere (Sanders, 1968; Gage and Tyler, 1991), but remain mostly unknown because of extreme conditions in sampling techniques. New insights into deep-sea biodiversity are thus necessary in order to better understand the grade of stability and vulnerability of these environments (Merrett and Haedrich, 1997), but should focus on largescale analyses (Bianchi and Morri, 2000) dealing, for example, with a whole enclosed deep-sea environment.

The Mediterranean Sea is an optimal environment for studying the patterns of biodiversity for several reasons. First of all, it is an enclosed sea with limited connection with the outer Atlantic ocean. Its constant 
deep water temperature of between 13 and $14^{\circ} \mathrm{C}$ and its high coastal input related to its closeness to land (Tyler, 2003; Sardà et al., 2004a) make it a natural laboratory for studying the factors involved in determining community diversity. Fishing pressure typically reaches depths of 800-900 $\mathrm{m}$ in the western basin, while in the rest of the Mediterranean it barely reaches $600 \mathrm{~m}$ (Mytilineou et al., 2005; Sardà et al., 2009). In addition, a trawling ban is in effect in the whole basin below 1000 $m$ depth - a unique situation at present for an enclosed sea (EC Regulation 1967/2006), allowing us to study quasi-pristine environments. Below these depths direct human impact is reduced to a minimum and is usually caused by litter discharge (Galil et al., 1995), whose impact has not yet been clarified.

Most bathyal and abyssal soft bottoms-with the exception of chemosynthetic habitats such as hydrothermal vents and cold seeps_-are driven by organic matter input from the upper strata (Smith et al., 2008; Corliss et al., 2009; Lampadariou et al., 2009). The case of the Mediterranean is unique, because it is a semi-enclosed sea that has a gradient of decreasing availability of organic matter from west to east (Bethoux et al., 1999; Danovaro et al., 1999). The Levantine deep basin, known to be generally highly oligotrophic (Azov, 1991; Psarra et al., 2000; Danovaro et al., 2001), also shows a clear scarcity of all size classes of benthic fauna (Danovaro et al., 1999; Basso et al., 2004; Lampadariou et al., 2009; Danovaro et al., 2010).

In the western Mediterranean basin, a series of studies have been conducted regarding the deep-sea megafaunal assemblages down to $2800 \mathrm{~m}$ depth (Abelló et al., 1988; Cartes and Sardà, 1993; Stefanescu et al., 1993; Sardà et al., 1994; Moranta et al., 1998; Carrassón and Cartes, 2002; D'Onghia et al., 2004b), but addressing only fishes and crustaceans. Few studies have dealt with non-crustacean invertebrates (Ramírez-Llodra et al., 2008; Ramírez-Llodra et al., 2010). Regarding the central basin, information about lower slope megafauna is scarce as studies conducted until now have focused on the upper and middle slope communities, and addressed only particular assemblages such as fishes (D'Onghia et al., 1998; Mytilineou et al., 2005) and crustaceans (Company et al., 2004; Politou et al., 2005). Concerning the whole Mediterranean Sea, studies conducted to date have shown the existence of a depth zonation of megafauna on the continental slope (Pérès, 1985; D’Onghia et al., 1998; Moranta et al., 1998; D'Onghia et al., 2004b), with peaks of biomass and abundance around $1200 \mathrm{~m}$ depth and a subsequent fall in ecological indicators below $1500 \mathrm{~m}$ depth (for a review, see Sardà et al., 2009). Another branch of studies, particularly carried out in the western Mediterranean, have dealt with the diet (Carrassón et al., 1997; Carrassón and Matallanas, 1998, 2001, 2002) and biology (Stefanescu et al., 1992; Massutí et al., 1996; Morales-Nin et al., 1996a, b) of the main fish species of the continental slope, as well as the biology of de- capod crustaceans (Company and Sardà, 2000; Puig et al., 2001; Company et al., 2003).

During the DESEAS trans-Mediterranean cruise conducted in 2001, benthic megafauna, including fishes and invertebrates of the western Balearic, western Ionian and eastern Ionian basins, was sampled (Sardà et al., 2004b). A series of community structure and distribution studies were conducted and published on fishes and crustaceans (Company et al., 2004; D'Onghia et al., 2004b; Sion et al., 2004), but the less abundant non-crustacean invertebrate species were not included in any of the subsequent analyses and detailed biodiversity studies of the whole megafauna community were not carried out. The objective of the present paper is to describe biodiversity patterns of whole benthic megafauna on Mediterranean continental slopes and deep basins, discussing the results in the light of recent advances in deep Mediterranean trophic characteristics.

\section{MATERIALS AND METHODS}

Megafaunal samples were collected during a transMediterranean sampling cruise carried out in June 2001, in the framework of the DESEAS project (Sardà et al., 2004b), aboard the R/V García del Cid (Fig. 1). A Otter-trawl Maireta system (OTMS) was used as sampling gear (Sardà et al., 1998). A total of 26 trawls were conducted at depths ranging from 600 to $4000 \mathrm{~m}$ (Table 1): seven in the western Balearic basin, eight in the western Ionian Sea, nine in the eastern Ionian Sea, and two in the central abyssal plain. The OTMS is a benthic trawling net fitted with two divergent doors and a single warp cable. The total net length is $25 \mathrm{~m}$, with a cod-end mesh size of $40 \mathrm{~mm}$ and an outer cover of $12 \mathrm{~mm}$. Bridles of $67 \mathrm{~m}$ length were used. A SCANMAR system was used to estimate the mean net opening geometry during the trawl, showing a horizontal opening of $12.7 \pm 1.4 \mathrm{~m}$. As the SCANMAR system can only operate down to $1200 \mathrm{~m}$ depth, the same value was used for deeper trawls, considering that no technical changes were made to the sampler during the cruise. Vertical opening was estimated at $1.4 \mathrm{~m}$, with substantially less variability due to the net building characteristics (Sardà et al., 1998).

Every megafaunal individual was identified to species level, counted and wet-weighted $( \pm 1 \mathrm{~g})$. A faunal list was compiled, along with the total catch abundance of each species by sampling zone and the depth range of occurrence. The SCANMAR data were used to standardize abundance (number $/ \mathrm{km}^{2}$ ) and biomass ( $\mathrm{kg} /$ $\mathrm{km}^{2}$ ) data between trawls. Species richness, ShannonWiener H', Margalef's richness index and Pielou's evenness index were calculated for each trawl (Shannon, 1948; Margalef, 1958; Pielou, 1966; Magurran, 2004). When replicate samples per depth were available, the mean Shannon index values were calculated. Similarity of community structure among samples from all geographic areas and depths was visualized 


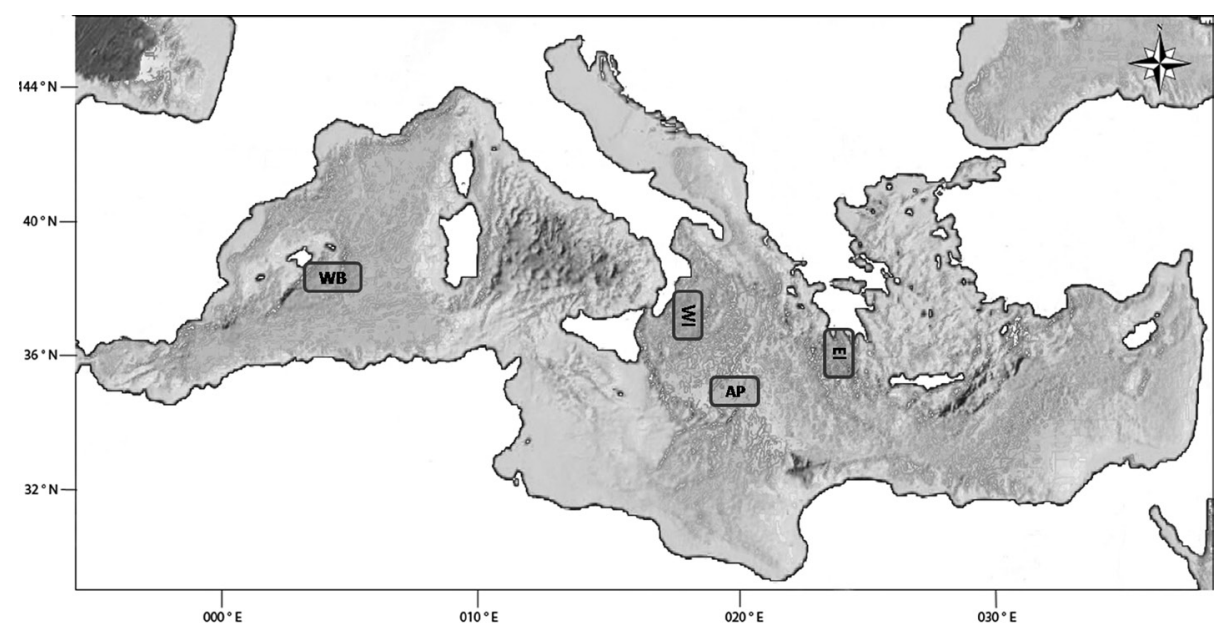

FIG. 1. - Map of Mediterranean Sea, including the sampled areas: western Balearic (WB), western Ionian (WI), eastern Ionian (EI) and the central abyssal plain (AP).

with a non-metric multidimensional scaling (MDS), after abundance data had been $\log (\mathrm{X}+1)$ transformed. This analysis is an iterative procedure that minimizes the difference between ranked Bray-Curtis similarity values and 2-dimensional distance between each pair of samples. MDS analysis was reiterated 1000 times with a minimum stress value of 0.01 , and was applied to all samples from all depths and geographic areas.

To assess what species mostly caused dissimilarities between trawls, the two-way similarity percentages (SIMPER) non-parametric statistical routine was conducted, using "depth" and "area" as crossed factors and the Bray-Curtis measure as similarity (Clarke and Warwick, 2001). All diversity analyses were conducted using the PRIMER-E 6 software package.

\section{RESULTS}

A total of 102 megafaunal species belonging to 5 phyla were collected and identified (Table 2). Of these, 15 species $(14,7 \%)$ were non-crustacean invertebrates belonging to the phyla Mollusca (classes Cephalopoda and Gastropoda), Echinodermata, Brachiopoda and Chordata (represented by the pyrosomatid tunicate Pyrosoma atlanticum). The most speciose groups were Actinopterygii ( $45 \%$ of species), crustaceans $(32.4 \%$ of species) and Chondrichthyes (7.8\% of species).

Multidimensional scaling analysis calculated a stress value of 0.1 , which indicates an acceptable grade of ordination with little risk of misinterpretation (Clarke and Warwick, 2001). The plot suggests a

TABLE 1. - List of analyzed trawls with time and spatial characteristics.

\begin{tabular}{|c|c|c|c|c|c|c|c|}
\hline \multirow[t]{2}{*}{ Area } & \multirow[t]{2}{*}{ Haul } & \multirow[t]{2}{*}{ Date } & \multirow[t]{2}{*}{ Mean depth (m) } & \multicolumn{2}{|c|}{ Start position } & \multicolumn{2}{|c|}{ End position } \\
\hline & & & & Latitude N & Longitude E & Latitude $\mathrm{N}$ & Longitude $\mathrm{E}$ \\
\hline \multirow[t]{7}{*}{ Balearic Sea } & W600 & 07.06 .2001 & 650 & $38^{\circ} 36.51$ & $001^{\circ} 53.29$ & $38^{\circ} 39.27$ & $001^{\circ} 55.12$ \\
\hline & W800 & 08.06 .2001 & 800 & $38^{\circ} 30.13$ & $001^{\circ} 49.12$ & $38^{\circ} 28.25$ & $001^{\circ} 46.80$ \\
\hline & W1000 & 08.06 .2001 & 1000 & $38^{\circ} 28.47$ & $001^{\circ} 53.20$ & $38^{\circ} 26.44$ & $001^{\circ} 50.41$ \\
\hline & W1200 & 06.06 .2001 & 1230 & $40^{\circ} 48.30$ & $001^{\circ} 48.13$ & $40^{\circ} 47.28$ & $001^{\circ} 45.12$ \\
\hline & W1500 & 08.06 .2001 & 1500 & $38^{\circ} 17.15$ & $001^{\circ} 48.10$ & $38^{\circ} 18.16$ & $001^{\circ} 44.30$ \\
\hline & W2500 & 09.06 .2001 & 2500 & $38^{\circ} 15.57$ & $002^{\circ} 21.10$ & $38^{\circ} 21.24$ & $002^{\circ} 23.43$ \\
\hline & W2800 & 10.06 .2001 & 2800 & $38^{\circ} 04.10$ & $005^{\circ} 27.40$ & $38^{\circ} 05.24$ & $005^{\circ} 34.60$ \\
\hline \multirow[t]{8}{*}{ Western Ionian Sea } & C600 & 14.06 .2001 & 600 & $38^{\circ} 18.50$ & $016^{\circ} 37.28$ & $38^{\circ} 17.37$ & $016^{\circ} 33.51$ \\
\hline & $\mathrm{C} 800$ & 14.06 .2001 & 800 & $38^{\circ} 16.56$ & $016^{\circ} 37.32$ & $38^{\circ} 18.01$ & $016^{\circ} 40.43$ \\
\hline & C1000 & 15.06 .2001 & 1000 & $38^{\circ} 15.11$ & $016^{\circ} 34.44$ & $38^{\circ} 15.25$ & $016^{\circ} 40.13$ \\
\hline & C1200 & 18.06 .2001 & 1200 & $32^{\circ} 13.58$ & $016^{\circ} 35.17$ & $38^{\circ} 13.92$ & $016^{\circ} 37.51$ \\
\hline & $\mathrm{C} 1500$ & 15.06 .2001 & 1500 & $38^{\circ} 10.16$ & $016^{\circ} 38.34$ & $38^{\circ} 12.17$ & $016^{\circ} 44.17$ \\
\hline & $\mathrm{C} 1500$ & 17.06 .2001 & 1500 & $38^{\circ} 12.70$ & $010^{\circ} 43.52$ & $38^{\circ} 10.33$ & $016^{\circ} 40.40$ \\
\hline & $\mathrm{C} 1700$ & 18.06 .2001 & 1700 & $38^{\circ} 06.13$ & $016^{\circ} 42.10$ & $38^{\circ} 08.17$ & $016^{\circ} 48.80$ \\
\hline & C2000 & 16.06 .2001 & 2000 & $37^{\circ} 42.18$ & $016^{\circ} 40.70$ & $37^{\circ} 39.40$ & $016^{\circ} 35.40$ \\
\hline \multirow[t]{9}{*}{ Eastern Ionian Sea } & E600 & 22.06 .2001 & 600 & $36^{\circ} 47.46$ & $022^{\circ} 04.29$ & $36^{\circ} 50.32$ & $022^{\circ} 04.10$ \\
\hline & E600 & 26.06 .2001 & 600 & $36^{\circ} 47.43$ & $022^{\circ} 04.28$ & $36^{\circ} 51.40$ & $022^{\circ} 03.55$ \\
\hline & E800 & 22.06 .2001 & 800 & $36^{\circ} 51.52$ & $022^{\circ} 06.16$ & $36^{\circ} 49.06$ & $022^{\circ} 06.16$ \\
\hline & E800 & 26.06 .2001 & 800 & $36^{\circ} 51.39$ & $022^{\circ} 06.12$ & $36^{\circ} 48.80$ & $022^{\circ} 06.17$ \\
\hline & E1100 & 24.06 .2001 & 1100 & $36^{\circ} 44.49$ & $022^{\circ} 07.32$ & $36^{\circ} 45.59$ & $022^{\circ} 10.58$ \\
\hline & E1300 & 22.06 .2001 & 1300 & $36^{\circ} 42.52$ & $022^{\circ} 10.25$ & $36^{\circ} 39.16$ & $022^{\circ} 10.55$ \\
\hline & $\mathrm{E} 1700$ & 25.06 .2001 & 1700 & $36^{\circ} 24.60$ & $022^{\circ} 14.60$ & $36^{\circ} 19.51$ & $022^{\circ} 14.90$ \\
\hline & E2200 & 23.06 .2001 & 2200 & $36^{\circ} 32.17$ & $022^{\circ} 01.46$ & $36^{\circ} 32.32$ & $022^{\circ} 09.83$ \\
\hline & E2600 & 23.06 .2001 & 2600 & $36^{\circ} 21.50$ & $022^{\circ} 00.60$ & $36^{\circ} 20.32$ & $021^{\circ} 54.39$ \\
\hline \multirow[t]{2}{*}{ Central abyssal plain } & A3300 & 02.07 .2001 & 3300 & $36^{\circ} 08.21$ & $016^{\circ} 32.90$ & $36^{\circ} 10.24$ & $016^{\circ} 24.80$ \\
\hline & A4000 & 01.07 .2001 & 4000 & $35^{\circ} 41.51$ & $017^{\circ} 47.00$ & $35^{\circ} 41.07$ & $017^{\circ} 37.80$ \\
\hline
\end{tabular}


TABLE 2. - Species list of demersal megafauna sampled in the DESEAS cruise, along with their total collected abundance in each of the four samples areas, and their depth range of catch.

\begin{tabular}{|c|c|c|c|c|c|c|c|c|c|}
\hline PHYLUM Family & Species & \multicolumn{2}{|c|}{$\begin{array}{c}\text { Western Balearic } \\
\mathrm{N} \mathrm{km}^{-2} \text { Depth }(\mathrm{m})\end{array}$} & \multicolumn{2}{|c|}{$\begin{array}{c}\text { Western Ionian } \\
\mathrm{N} \mathrm{km}^{-2} \text { Depth }(\mathrm{m})\end{array}$} & \multicolumn{2}{|c|}{$\begin{array}{l}\text { Eastern Ionian } \\
\mathrm{N} \mathrm{km}^{-2} \text { Depth }(\mathrm{m})\end{array}$} & \multicolumn{2}{|c|}{$\begin{array}{l}\text { Central abyssal plain } \\
\mathrm{N} \mathrm{km}^{-2} \text { Depth (m) }\end{array}$} \\
\hline \multicolumn{10}{|l|}{ MOLLUSCA } \\
\hline \multirow[t]{2}{*}{ Octopodidae } & Bathypolipus sponsalis & 34.9 & 1230 & & & & & & \\
\hline & Illex coindetii & & & & & 11.0 & 600 & & \\
\hline \multirow{2}{*}{$\begin{array}{l}\text { Enoploteuthidae } \\
\text { Histioteuthidae }\end{array}$} & Abralia veranyi & & & & & 11.6 & 1700 & & \\
\hline & $\begin{array}{l}\text { Histioteuthis bonnelli } \\
\text { Histioteuthis reversa }\end{array}$ & $\begin{array}{l}11.6 \\
13.2\end{array}$ & $\begin{array}{l}1230 \\
600\end{array}$ & 81.7 & $600-1200$ & & & & \\
\hline \multirow{2}{*}{$\begin{array}{l}\text { Ommastrephidae } \\
\text { Sepiolidae }\end{array}$} & Todarodes sagittatus & 42.2 & $600-800$ & & & & & & \\
\hline & $\begin{array}{l}\text { Heteroteuthis dispar } \\
\text { Neorossia caroli }\end{array}$ & & & $\begin{array}{c}49.9 \\
9.6\end{array}$ & $\begin{array}{l}1200 \\
1500\end{array}$ & $\begin{array}{l}24.1 \\
46.4\end{array}$ & $\begin{array}{l}600-800 \\
600\end{array}$ & & \\
\hline Aporrhaididae & Aporrhais serresiana & & & 55.9 & 800 & 757.0 & $600-800$ & & \\
\hline Naticidae & Natica sp. & & & 18.6 & 800 & & & & \\
\hline Cymbuliidae & Cymbulia peronii & & & 105.5 & 600 & & & & \\
\hline BRACHIOPODA & & & & & & & & & \\
\hline $\begin{array}{l}\text { Terebratulidae } \\
\text { PTHAPA }\end{array}$ & Gryphus vitreus & & & & & 131.5 & $600-800$ & & \\
\hline $\begin{array}{l}\text { ARTHROPODA } \\
\text { Phronimoidea }\end{array}$ & & & & & & & & & \\
\hline $\begin{array}{l}\text { Phronimoidea } \\
\text { Aristeidae }\end{array}$ & $\begin{array}{l}\text { Phronima sedentaria } \\
\text { Aristaeomornha foliacea }\end{array}$ & 263 & 600 & 18.6 & $\begin{array}{l}800 \\
600\end{array}$ & 109268 & $8600-1100$ & & \\
\hline & Aristeus antennatus & 3473.7 & $600-2800$ & 1456.5 & $600-2000$ & 1381.0 & $600-1700$ & 11.1 & 3300 \\
\hline Benthesicymidae & Gennadas elegans & 82.6 & $800-2800$ & 446.6 & $800-1500$ & 62.0 & $800-2200$ & & \\
\hline Crangonidae & Pontocaris lacazei & 13.2 & 600 & & & & & & \\
\hline & Pontophilus norvegicus & 23.2 & 1230 & 758.5 & $800-1700$ & & & & \\
\hline Galatheidae & Munida tenuimana & 223.7 & $600-1500$ & 57.3 & 1500 & & & & \\
\hline Geryonidae & Chaceon mediterraneus & 136.1 & $2500-2800$ & & & 19.1 & $1700-2200$ & 22.2 & 3300 \\
\hline & Geryon longipes & 792.4 & $600-1500$ & 13.2 & 600 & 15.4 & 1300 & & \\
\hline Goneplacidae & Goneplax rhomboides & & & 13.2 & 600 & & & & \\
\hline Hippolytidae & Ligur ensiferus & & & 11.4 & 1000 & & & & \\
\hline Homolidae & Paromola cuvieri & 48.6 & $800-1230$ & 43.2 & $600-1000$ & & & & \\
\hline Nematocarcinidae & Nematocarcinus exilis & 699.9 & $1500-2800$ & 496.8 & $1500-1700$ & 2657.7 & $1700-2600$ & 488.9 & $3300-4000$ \\
\hline Nephropidae & Nephrops norvegicus & & & & & 62.2 & 600 & & \\
\hline Oplophoridae & Acantephyra eximia & 1276.9 & $800-2800$ & 613.8 & $1000-2000$ & 1287.5 & $1300-2600$ & 333.3 & $3300-4000$ \\
\hline & Acantephyra pelagica & 90.9 & $100-2800$ & 132.1 & $1000-2000$ & & & 22.2 & $3300-4000$ \\
\hline Paguridae & Pagurus alatus & 14.5 & 800 & & & 39.4 & 800 & & \\
\hline Palaemonidae & Periclimenes granulatus & & & 18.6 & 800 & & & & \\
\hline Pandalidae & Plesionika martia & 462.4 & $600-800$ & 395.5 & 600 & 658.2 & $600-800$ & & \\
\hline & Plesionika acanthonotus & 554.2 & $600-1000$ & 82.8 & $600-1000$ & 369.0 & $600-800$ & & \\
\hline & Plesionika edwardsii & & & & & 13.4 & 600 & & \\
\hline Pasipaheidae & Plesionika gigliolii & 26.3 & 600 & & & 90.3 & 600 & & \\
\hline & Pasiphaea multidentata & 72.7 & $800-2500$ & $\begin{array}{l}71.9 \\
31.8\end{array}$ & $600-1500$ & 131 & 800 & & \\
\hline Peneidae & Parapenaeus longirostris & & & & & 44.0 & 600 & & \\
\hline Polychelidae & Polycheles typhlops & 65.3 & $800-1000$ & 1060.9 & $600-2000$ & 605.9 & $600-2200$ & & \\
\hline & Stereomastis sculpta & 319.5 & $2500-2800$ & & & & & & \\
\hline Portunidae & Bathynectes maravigna & 14.5 & 800 & 76.2 & $1000-1500$ & 30.8 & 1300 & & \\
\hline Processidae & Processa canaliculata & 13.2 & 600 & 26.4 & $600-1500$ & & & & \\
\hline Sergestidae & Sergestes arcticus & 13.2 & 600 & 53.1 & $600-1500$ & 13.4 & 600 & & \\
\hline & Sergestes corniculum & & & 87.7 & $600-1200$ & 39.6 & $600-1100$ & 22.2 & $3300-4000$ \\
\hline & Sergia robusta & 242.7 & $600-2800$ & 1851.0 & $600-2000$ & 184.0 & $800-2600$ & 33.3 & $3300-4000$ \\
\hline Xanthidae & Monodaeus couchi & 72.6 & 800 & & & 13.4 & 600 & & \\
\hline ECHINODERMATA & & & & & & & & & \\
\hline Astropectinidae & Astropecten irregularis & 23.2 & 1230 & & & & & & \\
\hline Echinidae & Echinus sp. & & & & & 12.9 & 800 & & \\
\hline CHORDATA & & & & & & & & & \\
\hline Pyrosomatidae & Pyrosoma atlanticum & & & & & 15.4 & 1300 & & \\
\hline Scyliorhinidae & Galeus melastomus & 1003.1 & $600-1500$ & 1085.6 & $600-1500$ & 917.5 & $600-1300$ & & \\
\hline Chimaeridae & Chimaera monstrosa & 13.2 & 600 & & & 26.0 & 800 & & \\
\hline Hexanchidae & Hexanchus griseus & & & & & 15.4 & 1300 & & \\
\hline Centrophoridae & Centrophorus granulosus & & & & & 13.3 & 1100 & & \\
\hline Dalatiidae & Dalatias licha & 26.1 & $800-1230$ & 99.9 & 1200 & 26.0 & 800 & & \\
\hline Etmopteridae & Etmopterus spinax & 150.9 & $800-1230$ & 194.2 & $600-1500$ & 219.8 & $600-2200$ & & \\
\hline Oxinotidae & Oxinotus centrina & & & 18.6 & 800 & & & & \\
\hline Somniosidae & Centroscymnus coelolepis & 237.8 & $1500-2800$ & & & & & & \\
\hline Halosuridae & Halosaurus ovenii & 7.7 & 2800 & & & & & & \\
\hline Notacanthidae & Notacanthus bonaparte & 11.6 & 1230 & 425.9 & $600-1500$ & 26.5 & 1100 & & \\
\hline & Polyacanthonotus rissoanus & & & & & 20.7 & $2200-2600$ & & \\
\hline Congridae & Conger conger & & & & & 13.4 & 600 & & \\
\hline Nettastomatidae & Nettastoma melanurum & 32.5 & 1000 & 376.5 & $600-1500$ & 307.5 & $600-1300$ & & \\
\hline Chlorophthalmidae & Chlorophthalmus agassizi & & & & & 1369.7 & 600 & & \\
\hline Ipnopidae & Bathypterois mediterraneus & 641.0 & $1000-2800$ & 3910.6 & $800-2000$ & 1500.9 & $800-2600$ & 22.2 & 3300 \\
\hline Paralepididae & Arctozenus risso & 10.8 & 1000 & & & & & & \\
\hline & Paralepis speciosa & & & 13.2 & 600 & 12.9 & 800 & & \\
\hline
\end{tabular}


TABLE 2 (cont.). - Species list of demersal megafauna sampled in the DESEAS cruise, along with their total collected abundance in each of the four samples areas, and their depth range of catch.

\begin{tabular}{|c|c|c|c|c|c|c|c|c|c|}
\hline \multirow{4}{*}{$\begin{array}{l}\text { PHYLUM Family } \\
\text { Trachichthyidae } \\
\text { Gadidae }\end{array}$} & \multirow{2}{*}{$\begin{array}{l}\text { Species } \\
\text { Hoplostethus mediterraneus }\end{array}$} & \multicolumn{2}{|c|}{$\begin{array}{l}\text { Western Balearic } \\
\mathrm{N} \mathrm{km}^{-2} \text { Depth }(\mathrm{m})\end{array}$} & \multicolumn{2}{|c|}{$\begin{array}{c}\text { Western Ionian } \\
\mathrm{N} \mathrm{km}^{-2} \text { Depth }(\mathrm{m})\end{array}$} & \multicolumn{2}{|c|}{$\begin{array}{l}\text { Eastern Ionian } \\
\mathrm{N} \mathrm{km}^{-2} \text { Depth }(\mathrm{m})\end{array}$} & \multicolumn{2}{|c|}{$\begin{array}{c}\text { Central abyssal plain } \\
\mathrm{N} \mathrm{km}^{-2} \text { Depth }(\mathrm{m})\end{array}$} \\
\hline & & 345.8 & $600-800$ & 349.9 & $600-800$ & 638.1 & $600-800$ & & \\
\hline & Micromesistius poutassou & 13.2 & 600 & 13.2 & 600 & 35.4 & 600 & & \\
\hline & Phycis blennoides & 531.9 & $600-1230$ & 145.1 & $600-1000$ & 121.4 & $600-800$ & & \\
\hline \multirow[t]{7}{*}{ Macrouridae } & Coelorinchus mediterraneus & 530.5 & $600-1500$ & 599.7 & $1200-1500$ & 30.8 & 1300 & & \\
\hline & Coryphaenoides guentheri & 295.0 & $2500-2800$ & 85.1 & $1500-1700$ & 54.6 & $1700-2600$ & & \\
\hline & Coryphaenoides mediterraneus & 569.5 & $1500-2800$ & 28.7 & 1500 & 94.9 & $1300-2600$ & 244.5 & $3300-4000$ \\
\hline & Hymenocephalus italicus & 307.2 & $600-800$ & 270.0 & $600-800$ & 96.6 & $600-800$ & & \\
\hline & Nezumia aequalis & 174.3 & 1230 & & & & & & \\
\hline & Nezumia sclerorhynchus & 1146.2 & $600-1500$ & 1229.8 & $600-1500$ & 443.7 & $600-1300$ & & \\
\hline & Trachyrincus scabrus & 697.1 & $800-1230$ & 125.5 & 1000 & & & & \\
\hline Merluccidae & Merluccius merluccius & & & & & 13.4 & 600 & & \\
\hline Moridae & Lepidion lepidion & 1160.0 & $1000-1500$ & 376.5 & $1200-1700$ & 115.8 & $1300-1700$ & & \\
\hline \multirow{6}{*}{ Myctophidae } & Mora moro & 765.0 & $800-1230$ & 1095.7 & $800-1200$ & 738.2 & $800-1300$ & & \\
\hline & Benthosema glaciale & & & & & 11.0 & 600 & & \\
\hline & Ceratoscopelus maderensis & 174.9 & $600-2500$ & & & 13.4 & 600 & & \\
\hline & $\begin{array}{l}\text { Diaphus metopoclampus } \\
\text { Hygophum benoiti }\end{array}$ & & & 18.6 & 800 & 251.1 & $600-800$ & & \\
\hline & Unidentified myctophid & & & & & & & 11.1 & 4000 \\
\hline & Lampanyctus crocodilus & 242.7 & $600-2500$ & 873.9 & $600-1500$ & 995.5 & $600-1300$ & 11.1 & 3300 \\
\hline Ophidiidae & Benthocometes robustus & 11.6 & 1230 & & & & & & \\
\hline Bythitidae & Cataetyx laticeps & 98.9 & $2500-2800$ & 65.9 & 2000 & 175.6 & $1700-2600$ & 11.1 & 3300 \\
\hline \multirow{3}{*}{$\begin{array}{l}\text { Alepocephalidae } \\
\text { Epigonidae }\end{array}$} & Alepocephalus rostratus & 5922.2 & $800-1500$ & & & & & & \\
\hline & Epigonus constanciae & & & & & 11.0 & 600 & & \\
\hline & $\begin{array}{l}\text { Epigonus denticulatus } \\
\text { Epigonus telescopus }\end{array}$ & 69.9 & $600-800$ & 22 & & & & & \\
\hline Trichiuridae & Lepidopus caudatus & 13.2 & 600 & & 1000 & & & & \\
\hline Sparidae & Pagellus bogaraveo & 26.3 & 600 & 11.4 & 1000 & & & & \\
\hline \multirow[t]{2}{*}{ Cynoglossidae } & Symphurus ligulatus & 13.2 & 600 & 52.7 & 600 & 23.9 & $600-800$ & & \\
\hline & Symphurus nigrescens & 14.5 & 800 & 290.0 & 600 & & & & \\
\hline \multirow[t]{2}{*}{ Scophthalmidae } & Lepidorhombus boscii & & & & & 92.7 & 600 & & \\
\hline & Lepidorhombus whiffiagonis & & & & & 13.4 & 600 & & \\
\hline \multirow[t]{2}{*}{ Scorpaenidae } & Helicolenus dactylopterus & 13.2 & 600 & & & 1741.8 & $600-800$ & & \\
\hline & Trachyscorpia cristulata & 28.1 & 1500 & & & & & & \\
\hline Triglidae & Peristedion cataphractum & & & & & 171.6 & $600-800$ & & \\
\hline Gonostomatidae & Cyclothone braueri & 20.8 & $600-2800$ & 49.9 & 1200 & & & & \\
\hline Sternoptychidae & Argyropelecus hemigymnus & 273.9 & $600-2800$ & 128.0 & $800-1500$ & & & & \\
\hline \multirow[t]{2}{*}{ Stomiidae } & Chauliodus sloani & 56.8 & $600-2500$ & 52.3 & $800-2000$ & 85.5 & $600-2200$ & & \\
\hline & Stomias boa & 14.5 & 800 & & & 138.1 & $600-1700$ & 33.3 & 4000 \\
\hline
\end{tabular}

bathymetric pattern of species distribution from 600 to $4000 \mathrm{~m}$ depth, as shown in Figure 2.

Across the whole dataset, Margalef's richness index (Fig. 3) shows a significant decrease with depth $\left(\mathrm{R}^{2}=\right.$ $0.746, \mathrm{p}<0.001, \mathrm{n}=26)$. Non-linear regression found a negative exponential relation:

$$
S R=4.483 e^{-0.0005 D},
$$

where SR is Margalef's richness index and D is depth (m).

Negative linear correlations between species richness and depth were found for each of the three zones considered separately (Fig. 4). The slopes of the regression lines were higher for the two Ionian sea zones than for the Balearic basin, indicating a steeper decrease in species diversity with depth in the central basin than in the western zone. Margalef index ranged from a maximum of 3.66 at $600 \mathrm{~m}$ depth in the eastern Ionian to a minimum of 0.92 at $2600 \mathrm{~m}$ depth in the same zone.

Values of Pielou's evenness index are plotted against depth for the three sampling zones in Figure 4. In the western Balearic basin, values of Pielou's index for the deepest depths were higher than the shallowest ones, with an outlier at $1230 \mathrm{~m}$, although no significant

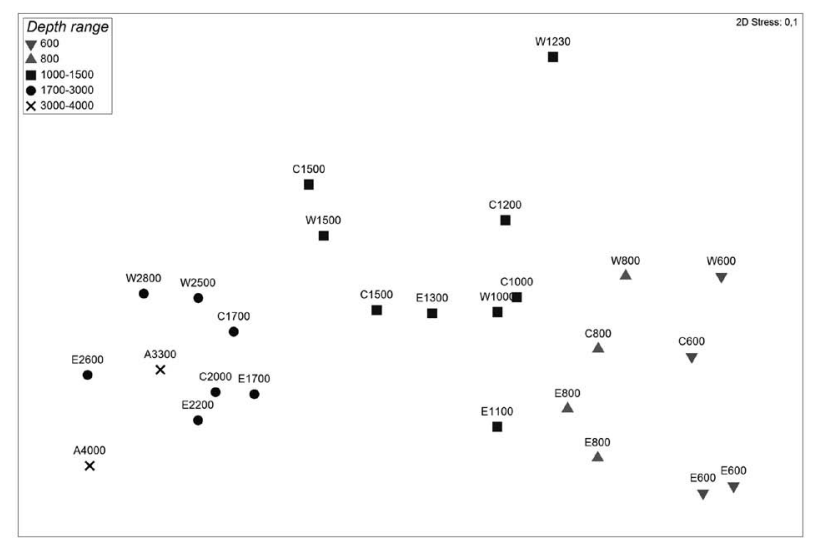

FIG. 2. - Multidimensional scaling plot of the whole abundance dataset. Points are pooled into depth-range groups, for $600-650 \mathrm{~m}$ (downward-pointing triangles), $800 \mathrm{~m}$ (upward-pointing triangles), 1000-1500 m (squares), 1700-3000 m (circles), and abyssal plain depths at 3300 and $4000 \mathrm{~m}$ (crosses). The code letter represents the sampling zone: western Balearic (W), western Ionian (C), eastern Ionian (E) and central abyssal plain (A).

trend was observed. A similar pattern was present in the western Ionian, with a decrease in the index at 1500 and $1700 \mathrm{~m}$ depth. In the eastern Ionian Sea, the even- 


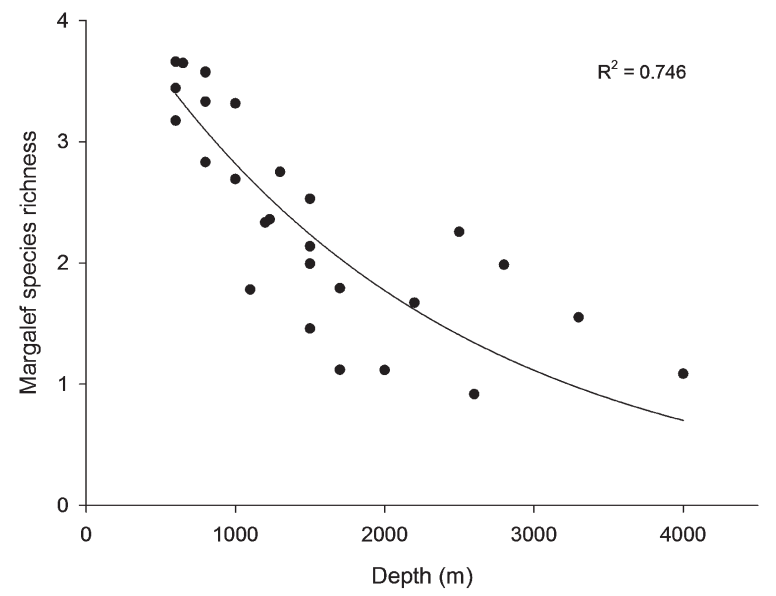

FIG. 3. - Margalef species richness index for the whole studied area (western and central Mediterranean basins).

Western Balearic
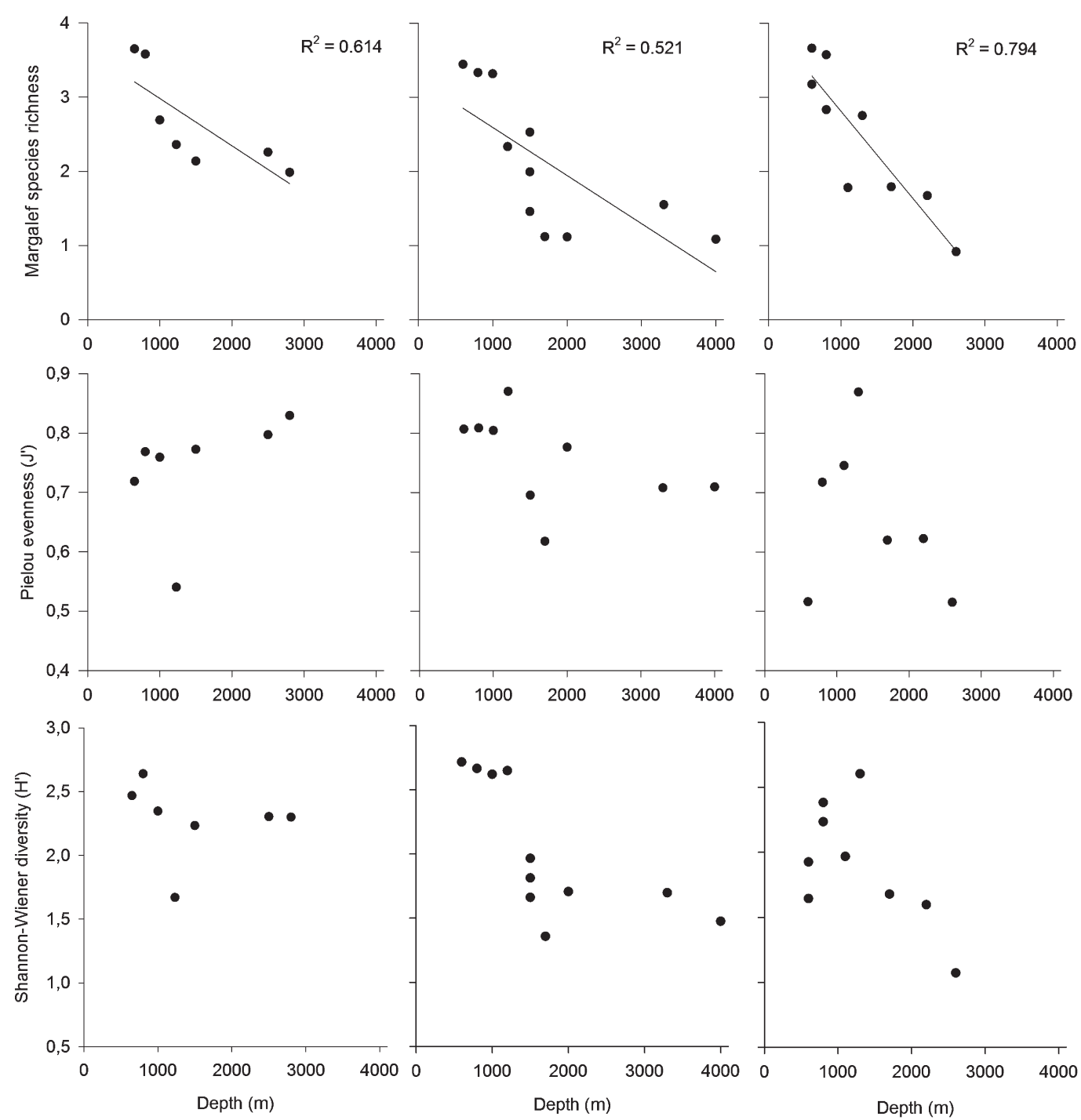

FIG. 4. - Margalef species richness, Pielou evenness and Shannon-Wiener diversity indices for the western Balearic Sea, the western Ionian sea (including the two central abyssal plain samples), and the eastern Ionian Sea. Linear regression lines are shown only when significant $(p<0.05)$. 

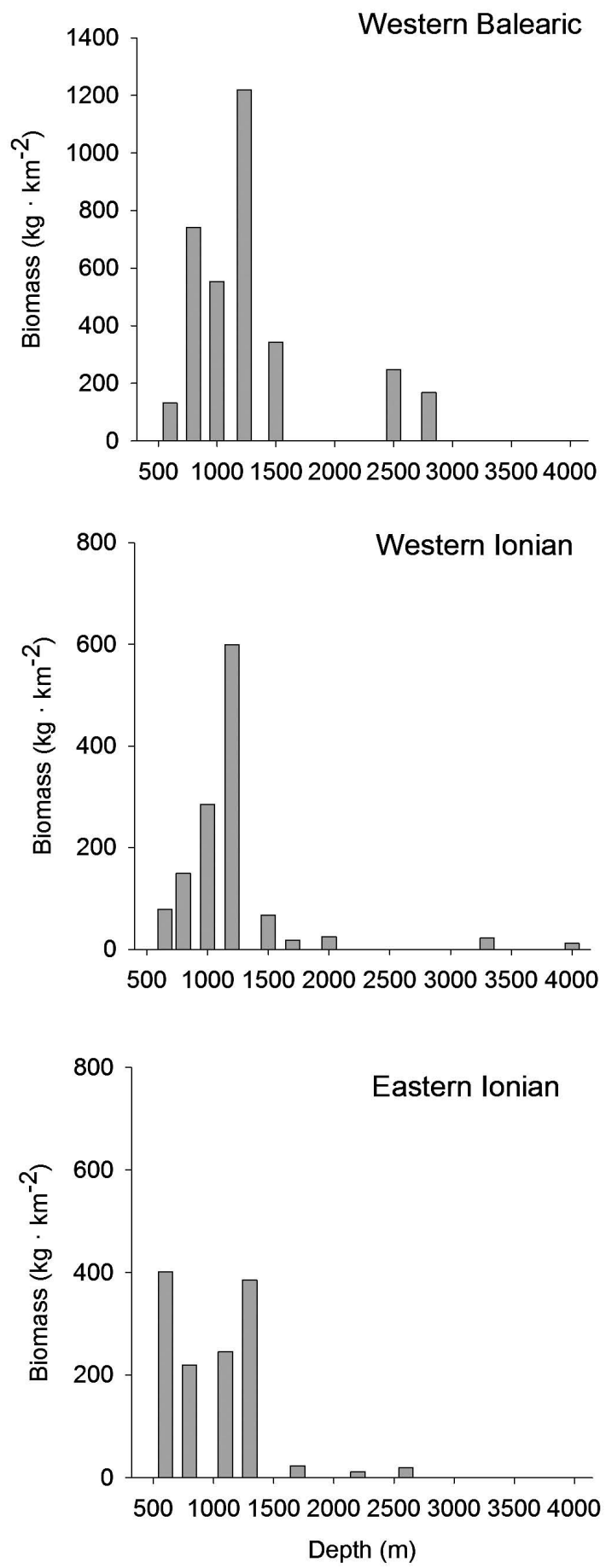

FIG. 5. - Standardized values of total catch biomass for the western Balearic Sea, the western Ionian sea (including the two central abyssal plain samples), and the eastern Ionian Sea.

Total catch biomass in the three zones was higher at the shallower depths $(600-1230 \mathrm{~m})$ than at the deeper depths (Fig. 5). In the Balearic basin the biomass at 800-1000 m depth was dominated by the fishes Alepocephalus rostratus, Mora moro and the shark Galeus melastomus; at $1200 \mathrm{~m}$ a peak was present, mainly caused by $A$. rostratus, and at depths of 2500-2800
TABle 3. - Dissimilarities between the three geographic zones as calculated by the SIMPER statistical routine.

\begin{tabular}{lcc}
\hline Species & Dissimilarity / S.D. & Contrib. \% \\
\hline Western Balearic - western Ionian (average dissimilarity: & 51.17 ) \\
Alepocephalus rostratus & 1.42 & 6.24 \\
Polycheles typhlops & 1.65 & 4.31 \\
Geryon longipes & 1.53 & 4.13 \\
Coryphaenoides mediterraneus & 0.89 & 3.75
\end{tabular}

Western Balearic - eastern Ionian (average dissimilarity: 48.12) $\begin{array}{lll}\text { Alepocephalus rostratus } & 0.92 & 4.73\end{array}$ $\begin{array}{lll}\text { Polycheles typhlops } & 1.29 & 4.13\end{array}$ Centroscymnus coelolepis $\quad 0.94 \quad 3.85$ $\begin{array}{lll}\text { Aristeus antennatus } & 1.43 & 3.58\end{array}$

Western Ionian - eastern Ionian (average dissimilarity: 41.70) Acantephyra pelagica $\quad 1.11 \quad 4.64$ Coryphaenoides mediterraneus $\quad 0.95 \quad 4.26$ Polycheles typhlops $\quad 0.93 \quad 3.98$ $\begin{array}{lll}\text { Lepidion lepidion } & 0.83 & 3.95\end{array}$

$\mathrm{m}$ the shark Centroscymnus coelolepis dominated the biomass. The western Ionian sea showed a single peak of biomass at $1200 \mathrm{~m}$ due to a high retrieval of the fish M. moro and the sharks G. melastomus and Dalatias licha. Two peaks were present at 600 and $1300 \mathrm{~m}$ in the eastern Ionian, the shallower one caused mainly by the fish Helicolenus dactylopterus and the crustacean Aristaeomorpha foliacea, while the deeper one was produced by a concentration of M. moro. At the two abyssal stations, biomass was comparable with that at shallower depths (1700 and $2000 \mathrm{~m}$ ) in the same western Ionian zone.

According to SIMPER analysis, dissimilarity in community composition between the western Balearic basin, the western Ionian and the eastern Ionian across all depth ranges is mainly caused by the fishes $A$. rostratus and Coryphaenoides mediterraneus, the shark C. coelolepis and the decapods Aristeus antennatus, Polycheles typhlops and Geryon longipes (Table 3).

\section{DISCUSSION}

The paucity of robust biodiversity analyses in deep sea ecosystems is mainly caused by sampling difficulties that often result in low replicate numbers. The DESEAS sampling project provided a wide spectrum of novel data about deep-sea megafauna, making it possible to conduct new biodiversity analyses of the bathyal and abyssal megafauna communities as a whole along a longitudinal and bathymetric gradient (Sardà et al., 2004b).

Few non-crustacean invertebrates were retrieved, with molluscan cephalopods and gastropods making up the most part. The number of species in this group was considerably lower than that of deep-sea faunistic studies conducted in the northeastern Atlantic (Billett et al., 2001), where the dominance of non-crustacean invertebrates is well documented (Gage and Tyler, 1991). Integrating data with other types of gear that stay more in contact with the substrate (e.g. an Agassiz dredge) may help to clarify this point. 
Results from the multidimensional scaling routine showed a clear segregation by depth of benthic megafauna in the Mediterranean. The trawls at 600-800 m grouped together, confirming the middle slope subdivision (Pérès, 1985). Differences in this depth range between the western Balearic basin and the two study sites of the central basin were more marked than at the other depths. The depth range 1000-1700 m showed the highest dispersion between trawls, as was previously reported for fishes (Stefanescu et al., 1993; D'Onghia et al., 2004b) and crustacean assemblages (Maynou and Cartes, 2000; Company et al., 2004) in the Mediterranean. The present study included non-crustacean invertebrates in the analyses and this did not change the overall community structure pattern, suggesting that the megafaunal invertebrates follow the same pattern of depth zonation as the other taxa. The deepest assemblages at 1700-3000 m, along with the two trawls conducted at 3300 and $4000 \mathrm{~m}$ in the central Ionian abyssal plain, grouped together, indicating a reduction in depth influence below $1500 \mathrm{~m}$. There was no clear pattern in the MDS plot that could be interpreted as an influence of longitude, except at middle slope depths where samples showed a slight grouping by geographic area. This suggests that depth is a stronger driver than geographic area for community composition, as was previously pointed out for the eastern Mediterranean basin megafauna (Kallianiotis et al., 2000) and meiofauna (Lampadariou et al., 2009). Influence of depth alone as a physical factor in determining assemblage composition is currently a matter of discussion, and some authors propose that megafaunal zonation is mainly controlled by food availability (Jones et al., 2003; Tyler, 2003; Soltwedel et al., 2009). Coupling biodiversity studies with trophic analyses of key species in each basin may shed light on this result. As reported by Politou et al. (2004), temperature showed a slight increase in a westeast axis (12.8-13.9 in the western basin, 13.3-14.2 in the central Mediterranean), while salinity showed no differences between zones. It is generally known that the hydrology of the deep Mediterranean is relatively stable and dissolved oxygen is also believed not to be limiting, with values constantly above $4 \mathrm{mlO}_{2} / \mathrm{l}$ (Miller et al., 1970). Therefore, relating faunistic assemblages with available environmental data is currently difficult and further detailed abiotic studies are necessary.

A difference in equation fits for Margalef species richness was found between the whole dataset and the three zones considered separately. These differences could be caused by a relatively low number of samples. Further analyses with a larger dataset may bring to light patterns different from linear relations. Although Margalef index is more dependent than other diversity measures on sampling effort (Magurran, 2004), which in our case was higher at shallower stations, a negative linear correlation was found in all three basins, confirming the expected general reduction in megafaunal richness with depth (Gage and Tyler, 1991). The three zones exhibited a comparable species richness up to $1500 \mathrm{~m}$ depth, but after this limit only the western Mediterranean maintained a Margalef index of above 2.0, while in the Ionian basins (both western and eastern) values decreased rapidly. The Mediterranean sea is known to show a west-east gradient of decreasing productivity (Danovaro et al., 1999; Tselepides et al., 2004). It is therefore possible that higher surface productivity and coastal input of the western Balearic basin supply the necessary energy to sustain a diverse community down to deeper depths. Furthermore, the western Mediterranean is exposed to cyclic downslope dense shelf-water cascading events, which provide massive inputs of organic matter to bathyal and abyssal depths (Canals et al., 2006; Company et al., 2008). In the more oligotrophic Ionian Sea, organic matter reaching the lower slope and the deep basin is reduced, so there is a decrease in diversity early in the depth range.

Pielou's index of evenness expresses the degree of dissimilarity of the observed sample with a hypothetical situation of all equally abundant species. In the western Balearic Pielou's index showed a dramatic reduction at $1230 \mathrm{~m}$ depth, caused by the peak of abundance of the fish Alepocephalus rostratus. This dominance has been already pointed out in several studies (Stefanescu et al., 1993; Morales-Nin et al., 1996b; Sardà et al., 2009), and may be caused by specific trophic conditions (Carrassón and Matallanas, 1998) and by the occurrence of shelf-water cascading events that bring enriched water at these depths (Canals et al., 2006). A similar effect is present in the western Ionian at lower slope depths, and is caused by the spiderfish Bathypterois mediterraneus. The biology of this species is more adapted to the scarce trophic resources (Carrassón and Matallanas, 2001; D’Onghia et al., 2004a), although the exact reasons for this dominance of $B$. mediterraneus are, at present, not described.

In the two samples collected from abyssal depths, few species were collected: 11 at $3300 \mathrm{~m}$ and 8 at 4000 $\mathrm{m}$. In both cases, the catch composition was dominated by the macrourid Coryphaenoides mediterraneus and by the deep water shrimps Acantephyra eximia and Nematocarcinus exilis. These occurrences have already been described as the deepest records for these species (Company et al., 2004; D'Onghia et al., 2004b). Species evenness at these two depths was low compared to the rest of the western Ionian basin. Food scarcity has been shown to limit the presence of large-sized species, favouring settlement of small-sized species that can crop more efficiently on a broader range of preys (sensu Dayton and Hessler, 1972). C. mediterraneus and $N$. exilis had lower mean sizes than other species of macrourid fishes and decapod crustaceans that inhabit the deep Mediterranean bottoms, suggesting that these species may be the only ones that can efficiently inhabit these low-energy environments.

Regarding biomass, in the western Balearic basin the pattern obtained is in accordance with that of previous studies conducted in this basin (Moranta et al., 1998; Massutí et al., 2004). In the western Ionian sea, 
biomass values remain low down to the abyssal plain at $4000 \mathrm{~m}$ depth. Biomass flow in the eastern Ionian sea essentially follows the same pattern, with a biomass fall starting from $1700 \mathrm{~m}$, showing that, even with different diversity patterns, the three basins exhibit a consistent biomass decrease starting from lower slope depths.

According to SIMPER analysis, dissimilarity in community composition between the Balearic basin and the Ionian Sea (both western and eastern zones) is mainly caused by a few species of Actinopterygii and decapod crustaceans. A. rostratus has its distribution limited to the western Mediterranean basin (MoralesNin et al., 1996b; D’Onghia et al., 2004b) but the reasons for this spatial segregation are yet to be identified. The brachyuran crab Geryon longipes were found in the western Ionian and in the eastern Ionian in relatively low abundances. The trophic strategy of this species is less adapted to the scarcity of energy resources in the eastern basin (Kitsos et al., 2005), where another crab of the family Geryonidae, Chaceon mediterraneus, is beginning to be present. C. mediterraneus was also found in the western Balearic basin at depths of 2500 and $2800 \mathrm{~m}$. No occurrences of these two species at the same time were recorded, in agreement with another study which addressed the eastern basin megafauna (Jones et al., 2003).

The changes in megafaunal biodiversity across a geographical axis in the Mediterranean Sea have been partly addressed in this work, which has evaluated patterns of species substitution and biomass decline with depth and longitude. At depths of 1200-1500 m an interruption in the expected pattern of biodiversity across depth ranges takes place, with two distinct actinopterygian species- $A$. rostratus in the western basin and $B$. mediterraneus in the Ionian sea-dominating the abundance and biomass. This is an important matter for further exploration, with the hope of possibly extending the study to the whole longitudinal axis of the Mediterranean basin including the easternmost areas, where deep-sea megafauna sampling is at present very scarce. In addition, focused analyses to relate biodiversity to food availability and ecosystem functioning will provide essential information to better understand the observed community structure and diversity patterns in deep Mediterranean megafauna.

\section{ACKNOWLEDGEMENTS}

The authors wish to kindly thank the participants of the DESEAS project and the officers and crew of the R/V García del Cid. The DESEAS project was funded by EEC DG Fisheries Study Contract N. 2000/0039. ST is funded by a JAE-PRE-2008 grant from the CSIC, Spain.

\section{REFERENCES}

Abelló, P., F.J. Valladares and A. Castellón. - 1988. Analysis of the structure of decapod crustacean assemblages off the Catalan coast (North-West Mediterranean). Mar. Biol., 98: 39-49.
Azov, Y. - 1991. Eastern Mediterranean - a marine desert? Mar. Pollut. Bull., 23: 225-232.

Basso, D., J. Thomson and C. Corselli. - 2004. Indications of low macrobenthic activity in the deep sediments of the eastern Mediterranean Sea. Sci. Mar., 68(Suppl. 3): 53-62.

Bethoux, J.P., B. Gentili, P. Morin, E. Nicolas, C. Pierre and D. Ruiz-Pino. - 1999. The Mediterranean Sea: a miniature ocean for climatic and environmental studies and a key for the climatic functioning of the North Atlantic. Prog. Oceanogr., 44: $131-146$.

Bianchi, C.N. and C. Morri. - 2000. Marine biodiversity of the Mediterranean Sea: situation, problems and prospects for future research. Mar. Pollut. Bull., 40: 367-376.

Billett, D.S.M., B.J. Bett, A.L. Rice, M.H. Thurston, J. Galéron, M. Sibuet and G.A. Wolff. - 2001. Long-term change in the megabenthos of the Porcupine Abyssal Plain (NE Atlantic). Prog. Oceanogr., 50: 325-348.

Canals, M., P. Puig, X. Durrieu de Madron, S. Heussner, A. Palanques and J. Fabres. - 2006. Flushing submarine canyons. Nature, 444: 354-357.

Carrassón, M. and J.E. Cartes. - 2002. Trophic relationships in a Mediterranean deep-sea fish community: partition of food resources, dietary overlap and connections within the benthic boundary layer. Mar. Ecol. Prog. Ser., 241: 41-55.

Carrassón, M. and J. Matallanas. - 1998. Feeding habits of Alepocephalus rostratus (Pisces: Alepocephalidae) in the Western Mediterranean Sea. J. Mar. Biol. Ass. UK, 78: 1295-1306.

Carrassón, M. and J. Matallanas. - 2001. Feeding ecology of the Mediterranean spiderfish, Bathypterois mediterraneus (Pisces: Chlorophthalmidae), on the western Mediterranean slope. Fish. Bull., 99: 266-274.

Carrassón, M. and J. Matallanas. - 2002. Diets of deep-sea macrourid fishes in the western Mediterranean. Mar. Ecol. Prog. Ser., 234: $215-228$

Carrassón, M., J. Matallanas and M. Casadevall. - 1997. Feeding strategies of deep-water morids on the western Mediterranean slope. Deep-Sea Res. I, 44: 1685-1699.

Cartes, J.E. and F. Sardà. - 1993. Zonation of deep-sea decapod fauna in the Catalan Sea (Western Mediterranean). Mar. Ecol. Prog. Ser., 94: 27-34.

Clarke, K.R. and R.M. Warwick. - 2001. Change in marine communities: an approach to statistical analysis and interpretation. PRIMER-E Ltd., Plymouth, UK.

Company, J.B., P. Maiorano, A. Tselepides, C.-Y. Politou, W. Plaity, G. Rotllant and F. Sardà. - 2004. Deep-sea decapod crustaceans in the western and central Mediterranean Sea: preliminary aspects of species distribution, biomass and population structure. Sci. Mar., 68(Suppl. 3): 73-86.

Company, J.B., P. Puig, F. Sardà, A. Palanques, M. Latasa and R. Scharek. - 2008. Climate influence on deep sea populations. PLOS ONE. 3: e1431.

Company, J.B. and F. Sardà. - 2000. Growth parameters of deepwater decapod crustaceans in the Northwestern Mediterranean Sea: a comparative approach. Mar. Biol., 136: 79-90.

Company, J.B., F. Sardà, P. Puig, J.E. Cartes and A. Palanques. 2003. Duration and timing of reproduction in decapod crustaceans of the NW Mediterranean continental margin: is there a general pattern? Mar. Ecol. Prog. Ser., 261: 201-216.

Corliss, B.H., C.W. Brown, X. Sun and W.J. Showers. - 2009. Deep-sea benthic diversity linked to seasonality of pelagic productivity. Deep-Sea Res. I, 56: 835-841.

D’Onghia, G., D. Lloris, L. Sion, F. Capezzuto and M. Labropoulou. - 2004a. Observations on the distribution, population structure and biology of Bathypterois mediterraneus Bauchot, 1962 in three areas of the Mediterranean Sea. Sci. Mar., 68(Suppl. 3): 163-170.

D’Onghia, G., C.-Y. Politou, A. Bozzano, D. Lloris, G. Rotllant, L. Sion and F. Mastrototaro. - 2004b. Deep-water fish assemblages in the Mediterranean Sea. Sci. Mar., 68(Suppl. 3): 87-99.

D'Onghia, G., A. Tursi, P. Maiorano, A. Matarrese and M. Panza. - 1998. Demersal fish assemblages from the bathyal grounds of the Ionian Sea (middle-eastern Mediterranean). Ital. J. Zool., 65: $287-292$.

Danovaro, R., J.B. Company, C. Corinaldesi, G. D’Onghia, B.S. Galil, C. Gambi, A.J. Gooday, N. Lampadariou, G.M. Luna, C. Morigi, K. Olu, P. Polymenakou, E. Ramírez-Llodra, A. Sabbatini, F. Sardà, M. Sibuet and A. Tselepides. - 2010. Deep-sea 
biodiversity in the Mediterranean Sea: The known, the unknown, and the unknowable. PLoS ONE. 5(8): e11832.

Danovaro, R., A. Dell'Anno, M. Fabiano, A. Pusceddu and A. Tselepides. - 2001. Deep-sea ecosystem response to climate changes: the eastern Mediterranean case study. Trends Ecol. Evol. 16: 505-510.

Danovaro, R., A. Dinet, G. Duineveld and A. Tselepides. - 1999. Benthic response to particulate fluxes in different trophic environments: a comparison between the Gulf of Lions-Catalan Sea (western-Mediterranean) and the Cretan Sea (eastern-Mediterranean). Prog. Oceanogr., 44: 287-312.

Dayton, P.K. and R.R. Hessler. - 1972. Role of biological disturbance in maintaining diversity in the deep sea. Deep-Sea Res., 19: 199-208.

European Union. - Council Regulation (EC) No 1967/2006 of 21 December 2006, concerning management measures for the sustainable exploitation of fishery resources in the Mediterranean Sea. Official Journal of the European Union, L 409/11.

Gage, J.D. and P.A. Tyler. - 1991. Deep-sea biology: a natural history of organisms at the deep-sea floor. Cambridge University Press, Cambridge.

Galil, B.S., A. Golik and M. Türkay. - 1995. Litter at the bottom of the sea: a sea bed survey in the Eastern Mediterranean. Mar. Pollut. Bull., 30: 22-24.

Jones, E.G., A. Tselepides, P.M. Bagley, M.A. Collins and I.G. Priede. - 2003. Bathymetric distribution of some benthic and benthopelagic species attracted to baited cameras and traps in the deep eastern Mediterranean. Mar. Ecol. Prog. Ser., 251: 75-86.

Kallianiotis, A., K. Sophronidis, P. Vidoris and A. Tselepides. 2000. Demersal fish and megafaunal assemblages on the Cretan continental shelf and slope (NE Mediterranean): seasonal variation in species density, biomass and diversity. Prog. Oceanogr., 46: 429-455.

Kitsos, M.-S., S. Doulgeraki, A. Tselepides and A. Koukouras. 2005. Diet composition of the bathyal crabs, Chaceon mediterraneus Manning and Holthuis and Geryon longipes A. MilneEdwards (Decapoda, Geryonidae) collected at different depths in the eastern Mediterranean. Crustaceana, 78(2): 171-184.

Lampadariou, N., A. Tselepides and E. Hatziyanni. - 2009. Deepsea meiofaunal and foraminiferal communities along a gradient of primary productivity in the eastern Mediterranean Sea. Sci. Mar., 73 (2): 337-345.

Magurran, A.E. - 2004. Measuring biological diversity. Blackwell Science.

Margalef, R. - 1958. Information theory in ecology. Gen. Syst., 3: 36-71.

Massutí, E., J.D.M. Gordon, J. Moranta, S.C. Swan, C. Stefanescu and N.R. Merrett. - 2004. Mediterranean and Atlantic deep-sea fish assemblages: differences in biomass composition and sizerelated structure. Sci. Mar., 68(Suppl. 3): 101-115.

Massutí, E., B. Morales-Nin and D. Lloris. - 1996. Bathymetric distribution and recruitment patterns of Phycis blennoides (Pisces: Gadidae) from the slope of the northwestern Mediterranean. Sci. Mar., 60 (4): 481-488.

Maynou, F. and J.E. Cartes. - 2000. Community structure of bathyal decapod crustaceans off south-west Balearic Islands (western Mediterranean): seasonality and regional patterns in zonation. J. Mar. Biol. Ass. UK, 80: 789-798.

Merrett, N.R. and R.L. Haedrich. - 1997. Deep-sea demersal fish and fisheries. Chapman and Hall, London.

Miller, A.R., P. Tchernia and H. Charnock. - 1970. Mediterranean Sea Atlas of temperature, salinity, oxigen, profiles and data from cruises of R.V. Atlantis and R.V. Chain. Woods Hole Oceanographic Institution, Woods Hole, Massachussetts.

Morales-Nin, B., E. Massutí and C. Stefanescu. - 1996a. Bathymetric distribution and growth patterns of Bathypterois mediterraneus from the north-western Mediterranean Sea. J. Fish. Biol., 49(A): 276-288.

Morales-Nin, B., E. Massutí and C. Stefanescu. - 1996b. Distribution and biology of Alepocephalus rostratus from the Mediterranean Sea. J. Fish. Biol., 48: 1097-1112.

Moranta, J., C. Stefanescu, E. Massutí, B. Morales-Nin and D. Lloris. - 1998. Fish community structure and depth-related trends on the continental slope of the Balearic Islands (Algerian basin, western Mediterranean) Mar. Ecol. Prog. Ser., 171: 247-259.
Mytilineou, C., C.-Y. Politou, C. Papaconstantinou, S. Kavadas, G. D'Onghia and L. Sion. - 2005. Deep-water fish fauna in the Eastern Ionian Sea. Belg. J. Zool., 135: 229-233.

Pérès, J.M. - 1985. History of the Mediterranean biota and colonization of the depths. In: R. Margalef (ed.), Western Mediterranean, pp. 198-232. Pergamon Press, Oxford.

Pielou, E.C. - 1966. The measurement of diversity in different types of biological collections. J. Theor. Biol., 13: 131-144.

Politou, C.-Y., K. Kapiris, P. Maiorano, F. Capezzuto and J. Dokos. 2004. Deep-sea Mediterranean biology: the case of Aristaeomorpha foliacea (Risso, 1827) (Crustacea: Decapoda: Aristeidae). Sci. Mar., 68(Suppl. 3): 129-139.

Politou, C.-Y., P. Maiorano, G. D’Onghia and C. Mytilineou. 2005. Deep-water decapod crustacean fauna of the Eastern Ionian Sea. Belg. J. Zool., 135: 235-241.

Psarra, S., A. Tselepides and L. Ignatiades. - 2000. Primary productivity in the oligotrophic Cretan Sea (NE Mediterranean): seasonal and interannual variability. Prog. Oceanogr., 46: 187-204.

Puig, P., J.B. Company, F. Sardà and A. Palanques. - 2001. Responses of deep-water shrimp populations to intermediate nepheloid layer detachments on the Northwestern Mediterranean continental margin. Deep-Sea Res. I, 48: 2195-2207.

Ramírez-Llodra, E., M. Ballesteros, J.B. Company, L. Dantart and F. Sardà. - 2008. Spatio-temporal variations of biomass and abundance in bathyal non-crustacean megafauna in the Catalan Sea (North-western Mediterranean). Mar. Biol., 153: 297-309.

Ramírez-Llodra, E., J.B. Company, F. Sardà and G. Rotllant. - 2010. Megabenthic diversity patterns and community structure of the Blanes submarine canyon and adjacent slope in the Northwestern Mediterranean: a human overprint? Mar. Ecol., 31: 167-182.

Sanders, H.L. - 1968. Marine benthic diversity: a comparative study. Am. Nat., 102: 243-282.

Sardà, F., J.E. Cartes and J.B. Company. - 1994. Spatio-temporal variations in megabenthos abundance in three different habitats of the Catalan deep-sea (Western Mediterranean). Mar. Biol., 120: 211-219.

Sardà, F., J.E. Cartes, J.B. Company and A. Albiol. - 1998. A modified commercial trawl used to sample deep-sea megabenthos. Fish. Sci., 64: 492-493.

Sardà, F., A. Calafat, M. Flexas, A. Tselepides, M. Canals, M. Espino and A. Tursi. - 2004a. An introduction to Mediterranean deep-sea biology. Sci. Mar., 68(Suppl. 3): 7-38

Sardà, F., G. D'Onghia, C.-Y. Politou and A. Tselepides. - 2004b. Mediterranean deep-sea biology. Sci. Mar., 68(Suppl. 3): 1-204.

Sardà, F., J.B. Company, G. Rotllant and M. Coll. - 2009. Biological patterns and ecological indicators for Mediterranean fish and crustaceans below 1,000 m: a review. Rev. Fish Biol. Fish., 19: 329-347.

Shannon, C.H. - 1948. A mathematical theory of communication. Bell Sys. Tech. J., 27: 623-656.

Sion, L., A. Bozzano, G. D’Onghia, F. Capezzuto and M. Panza. 2004. Chondrichthyes species in deep waters of the Mediterranean Sea. Sci. Mar., 68(Suppl. 3): 153-162.

Smith, C.R., F.C. De Leo, A.F. Bernardino, A.K. Sweetman and P. Martinez Arbizu. - 2008. Abyssal food limitation, ecosystem structure and climate change. Trends Ecol. Evol., 23: 518-528.

Soltwedel, T., N. Jaeckisch, N. Ritter, C. Hasemann, M. Bergmann and M. Klages. - 2009. Bathymetric patterns of megafaunal assemblages from the arctic deep-sea observatory HAUSGARTEN. Deep-Sea Res. I, 56: 1856-1872.

Stefanescu, C., D. Lloris and J. Rucabado. - 1993. Deep-sea fish assemblages in the Catalan sea (western Mediterranean) below a depth of $1000 \mathrm{~m}$. Deep-Sea Res. I, 40: 695-707.

Stefanescu, C., J. Rucabado and D. Lloris. - 1992. Depth-size trends in western Mediterranean demersal deep-sea fishes. Mar. Ecol. Prog. Ser., 81: 205-213.

Tselepides, A., N. Lampadariou and E. Hatziyanni. - 2004. Distribution of meiobenthos at bathyal depths in the Mediterranean Sea. A comparison between sites of contrasting productivity. Sci. Mar., 68(Suppl. 3): 39-51.

Tyler, P.A. - 2003. Ecosystems of the world (Ecosystems of the Deep Ocean). Elsevier, Amsterdam.

Scient. ed.: D. Vaqué.

Received November 11, 2009. Accepted October 15, 2010.

Published online March 14, 2011. 